\title{
Arthroscopic Treatment of Infrapatellar Fat Pad Impingement between the Patella and Femoral Trochlea: Comparison of the Clinical Outcomes of Partial and Subtotal Resection
}

\author{
Young-Mo Kim, MD, PhD and Yong-Bum Joo, MD, PhD \\ Department of Orthopedic Surgery, Chungnam National University Hospital, Chungnam National University School of Medicine, Daejeon, Korea
}

Purpose: To compare the clinical outcomes of arthroscopic partial and subtotal resection of the impinged infrapatellar fat pad (IFP).

Materials and Methods: This study enrolled 55 patients with IFP impingement who underwent partial resection ( $\mathrm{n}=29$, $\mathrm{P}$ group) or subtotal resection ( $n=26, S$ group). Clinical outcomes at least 2 years postoperatively were evaluated using a visual analog scale (VAS) for pain, the International Knee Documentation Committee (IKDC) 2000 subjective knee score, and the Lysholm score.

Results: In the P group, the preoperative mean VAS, IKDC 2000, and Lysholm scores were 5.6 $\pm 0.61,47.5 \pm 7.41$, and 42.5 \pm 7.17 , respectively, which improved significantly to $1.4 \pm 0.63,70.9 \pm 6.15$, and $82.2 \pm 7.61$, respectively (all, $\mathrm{p} \leq 0.001$ ). In the $\mathrm{S}$ group, the preoperative mean VAS, IKDC 2000, and Lysholm scores were $5.7 \pm 0.43,47.7 \pm 9.09$, and $45.2 \pm 4.18$, respectively, which improved significantly to $1.8 \pm 0.77,71.9 \pm 9.33$, and $82.3 \pm 6.01$, respectively (all, $\mathrm{p} \leq 0.001$ ). There were no significant differences in any outcome measure between the groups at the final follow-up.

Conclusions: Arthroscopic resection of the impinged IFP between the patella and femoral trochlea resulted in favorable clinical outcomes regardless of whether the resection was partial or subtotal. Thus, partial resection can be an appropriate treatment option, considering partial resection was as effective as subtotal resection and retained more of the IFP.

Keywords: Fat pad, Infrapatellar, Impingement, Arthroscopy, Resection

\section{Introduction}

Infrapatellar fat pad (IFP) impingement, first described by Hof$\mathrm{fa}^{1)}$ in 1904, is an impingement syndrome caused by edema and swelling of the IFP between the patella and the femoral condyle.

Received April 25, 2018; Revised (1st) August 3, 2018; (2nd) August 10, 2018; Accepted August 16, 2018

Correspondence to: Yong-Bum Joo, MD, $\mathrm{PhD}$

Department of Orthopedic Surgery, Chungnam National University

Hospital, Chungnam National University School of Medicine, 282

Munhwa-ro, Jung-gu, Daejeon 35015, Korea

Tel: +82-42-338-2482, Fax: +82-42-338-2480

E-mail: longman76@hanmail.net

Source of funding: This research was supported by Chungnam National University Research Fund.

This is an Open Access article distributed under the terms of the Creative Commons Attribution Non-Commercial License (http://creativecommons.org/licenses/by-nc/4.0/) which permits unrestricted non-commercial use, distribution, and reproduction in any medium, provided the original work is properly cited.
The IFP is densely innervated ${ }^{2-4)}$, so impingement can cause acute or chronic sharp pain in the infrapatellar region ${ }^{5-7)}$. Constant irritation results in swelling and inflammation of the fat pad and injury to the patellar cartilage (Fig. 1).

Conservative treatment with non-steroidal anti-inflammatory drugs, physiotherapy, taping, and injections of local anesthetics and/or corticosteroids has been reported to relieve symptoms ${ }^{7-10)}$. When conservative strategies are not effective, surgery may be indicated $^{3)}$. Several studies have found that arthroscopic resection of the fat pad can be an effective treatment ${ }^{3,8,10-12}$. However, the extent of IFP resection that is required to achieve the best outcomes is unknown. The purpose of this study was to compare the clinical outcomes of patients undergoing arthroscopic partial and subtotal resection of the impinged IFP to identify the appropriate extent of resection. We hypothesized that (1) alleviation of symptoms would be expected with excision of only the impinged part of the IFP and (2) minimizing the amount resected would be most effective for postoperative pain relief because the IFP is a 
pain-sensitive tissue.

\section{Materials and Methods}

\section{Patient Selection and Diagnosis}

Collection of data in our registry was approved by our Institutional Review Board (No. CNUH 2015-11-020). All patients provided informed consent before participation. We followed consecutive 62 patients with IFP impingement treated by arthroscopic resection at our institution between October 2010 and October 2015. We diagnosed IFP impingement if there was a complaint of sharp pain in the infrapatellar region and two or more of following: (1) a positive Hoffa's test ${ }^{11,13,14)}$, (2) suspicious impingement of the IFP between the patella and femoral trochlea evident on magnetic resonance imaging (MRI) in the absence of

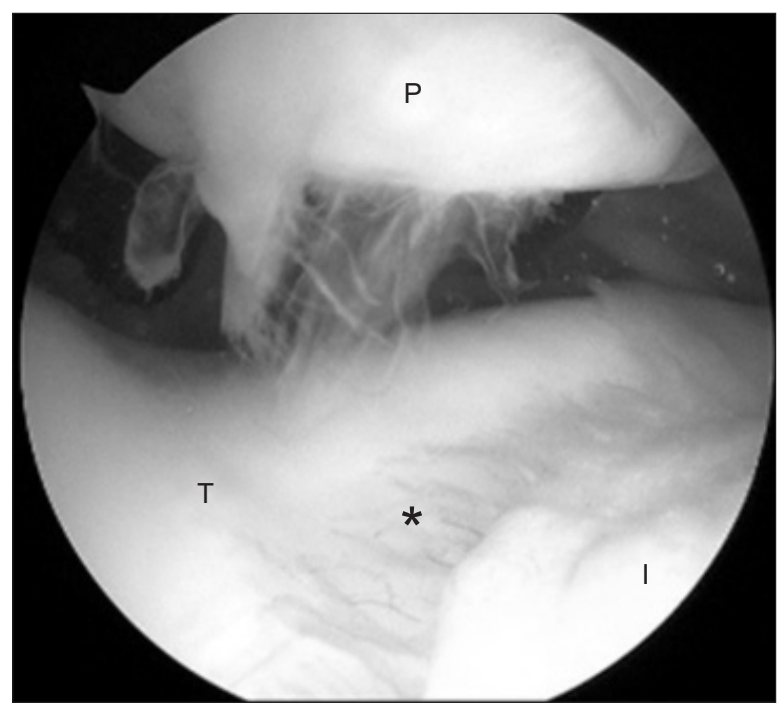

Fig. 1. Arthroscopic view showing the patellar cartilage injury due to infrapatellar fat pad (IFP) impingement. The IFP has a tongue-like protrusion between the patella and femoral trochlea. P: patella, T: trochlea, I: infrapatellar fat pad, asterisk: inflammation of the IFP. other intra-articular abnormalities, and (3) pain that was relieved by infiltration of lidocaine into the affected area. Surgery was undertaken in those patients who had consistent symptoms despite more than 3 months of conservative treatment.

We excluded patients with the following criteria: (1) meniscal or ligamentous lesions requiring concomitant treatment, (2) psychiatric problems, (3) advanced arthritic changes in the affected knee joint, (4) the presence of neurological disease, and (5) follow-up period of less than 2 years. Among the 62 patients, 7 patients were excluded. Five were lost to follow-up and two underwent surgery in the affected knee during the follow-up period (one for an anterior cruciate ligament rupture and the other for a meniscal tear). Finally, 55 patients were enrolled in this study. Arthroscopic partial resection of the IFP was performed in 29 patients ( $\mathrm{P}$ group) and subtotal resection was performed in 26

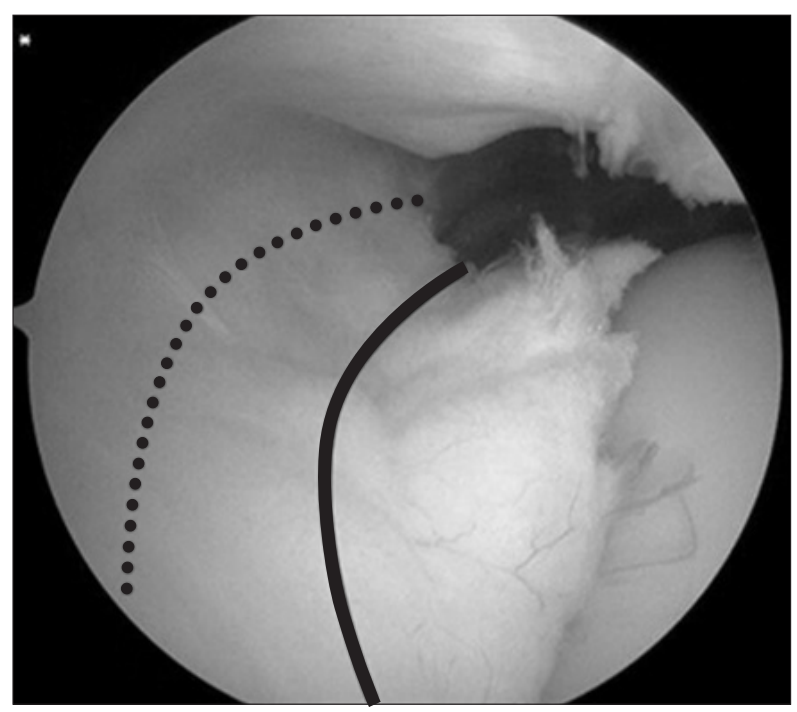

Fig. 2. Arthroscopic view showing the extent of partial and subtotal resection. The solid line indicates the margin of partial resection; the dotted line indicates the margin of subtotal resection.
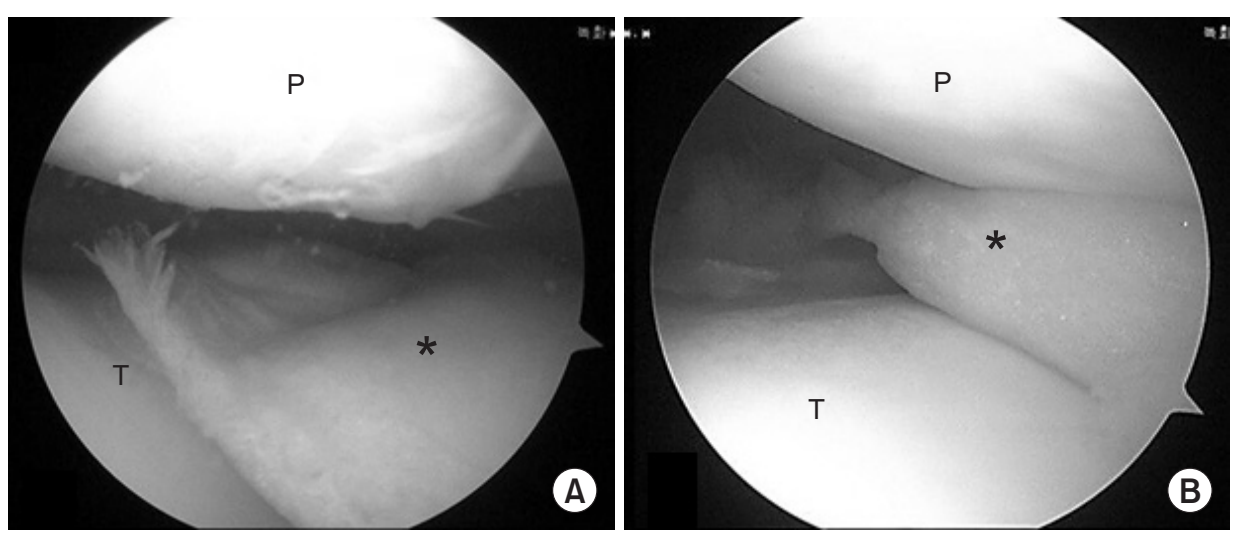

Fig. 3. Arthroscopic view with a $30^{\circ}$ arthroscope through the anterolateral portal of the right knee. (A) At $30^{\circ}$ knee flexion, the infrapatellar fat pad (asterisk) was observed. (B) When the knee was fully extended, the infrapatellar fat pad was trapped between the patellar undersurface and femoral trochlea. P: patella, T: trochlea, asterisk: infrapatellar fat pad. 


\section{Kim and Joo. Arthroscopic Resection of Infrapatellar Fat Pad Impingement}

patients (S group).

We classified the amount of fat pad resected as partial or subtotal. In a partial resection, only the impinged part of the IFP was excised, while in a subtotal resection, two-thirds of the IFP was excised, retaining the third at its base (Fig. 2).

\section{Surgical Technique}

All surgeries were performed under general anesthesia using a pneumatic tourniquet. We used the anterolateral (AL) or superolateral (SL) portal as a viewing portal and the anteromedial (AM) or SL portal as a working portal. The $30^{\circ}$ arthroscope was inserted through the AL portal and a routine arthroscopic examination of the whole knee joint was performed. Careful probing through the AM portal was performed to identify the other intraarticular lesions. Then the arthroscope was inserted through the SL portal. Viewing trough the SL portal, we assessed the impingement of the IFP between the patella and the femoral trochlea. Characteristically, when the knee was fully extended, the IFP was trapped between the patellar undersurface and femoral trochlea (Fig. 3). A motorized shaver was inserted through the AM portal to remove the impinged fat pad. While looking through the $\mathrm{AL}$ portal, the shaver was placed through the SL portal. After partial (Fig .4) or subtotal resection (Fig. 5) of the IFP, we checked if the fat pad would be impinged while range of motion of the knee. All surgeries were performed by one senior surgeon (YMK). From 2013, the resection technique was changed from subtotal resection to partial resection.

From the first postoperative day, range of motion exercises using a continuous passive motion device were started, and quadriceps muscle strengthening was performed several times daily. The weight bearing status was dependent on the individual condition, but all patients could bear full weight 7 days postoperatively.

\section{Clinical Evaluation}

Clinical data were collected preoperatively and the final followup. Three outcome measures were assessed in this study: 10-point visual analog scale (VAS) pain score (0, no pain; 5 , moderate pain; and 10, worst possible pain) ${ }^{15}$, the International Knee Documentation Committee (IKDC) 2000 subjective knee score ${ }^{16}$, and the Lysholm knee score ${ }^{17}$. We investigated complications related to surgery at the time of surgery and at the last follow-up.
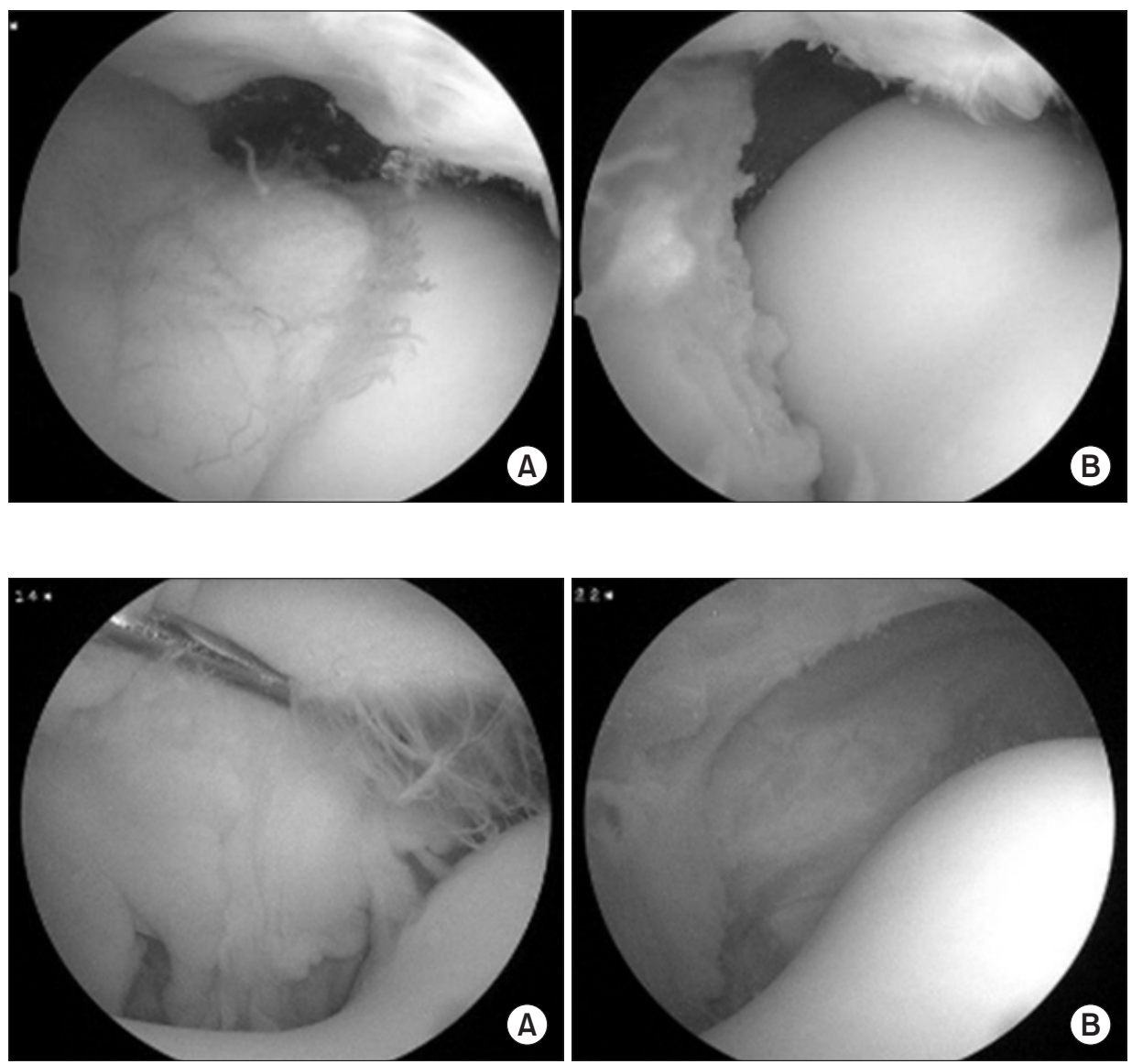

Fig. 4. Partial resection of the infrapatellar fat pad. (A) Before resection. (B) After resection.

Fig. 5. Subtotal resection of the infrapatellar fat pad. (A) Before resection. (B) After resection. 
Complications included neurovascular injury, infection, injury of the patellar tendon, and postoperative change of the patella height.

\section{Statistical Analysis}

All statistical analyses were performed with IBM SPSS ver. 23.0 (IBM Corp., Armonk, NY, USA) with a significance set at $\mathrm{p}<0.05$. We used the independent samples $t$-test and Fisher exact test to assess differences in the demographic and clinical characteristics. We used the independent sampled $t$-test and paired samples $t$-test to compare the preoperative and last follow-up data within the groups. Before the study, we calculated the necessary sample size. For the independent samples $t$-test, the statistical significance level of 0.05 , the power of 0.80 , the large effect size of 0.8 , and the applied allocation ratio of 1 were calculated. The calculated sample size was 26 for each group and 52 in total. At the same level, for the Fisher exact test, the calculated sample size was 18 for each group and 36 in total; for the paired samples $t$-test, a necessary sample size was 15 for each group and 30 in total. Therefore, our sample size was sufficient for statistical analysis: 55 patients in total including 29 patients in the $\mathrm{P}$ group and 26 patients in the $S$ group.

\section{Results}

Demographic and clinical characteristics of each group were not significantly different (Table 1). The mean final follow-up period was 32.5 months for the P group and 34.6 months for the $S$ group
( $\mathrm{p}=0.495)$. Preoperative mean VAS, IKDC 2000, and Lysholm scores were $5.6 \pm 0.61,47.5 \pm 7.41$, and $42.5 \pm 7.17$, respectively, in the P group, and $5.7 \pm 0.43,47.7 \pm 9.09$, and $45.2 \pm 4.18$, respectively, in the $S$ group. There were no significant differences between the two groups (Table 2). At the final follow-up, both groups showed a significant improvement in the clinical outcomes after surgery. Mean VAS, IKDC 2000, and Lysholm scores improved significantly to $11.4 \pm 0.63,70.9 \pm 6.15$, and $82.2 \pm 7.61$, respectively, in the P group, and to $1.8 \pm 0.77,71.9 \pm 9.33$, and $82.3 \pm 6.01$, respectively, in the $\mathrm{S}$ group (all $\mathrm{p} \leq 0.001$ ) (Table 2). There were no significant differences in any of the outcome measures between the groups at the final follow-up (Table 2). There were no complications related to the arthroscopic surgery immediately postoperatively and at the last follow-up.

\section{Discussion}

IFP impingement can be misdiagnosed as other intra-articular pathologies, including meniscal tears, patella-femoral pain syndrome, and patella tendinitis, as well as extra-articular pathologies ${ }^{7,8,18}$. It is important to take a rigorous approach to the diagnosis of IFP impingement by means of physical examination including Hoffa's test ${ }^{11}$, MRI, and interventional techniques such as intra-articular local anesthetic and/or corticosteroid injection $^{12,13)}$. Hoffa's test is difficult to conduct but is highly specific: with the patient lying down with their knee flexed, the examiner presses both thumbs along either side of the patellar tendon, just below the patella. The patient is then asked to extend the knee.

Table 1. Patient Demographic and Clinical Characteristics

\begin{tabular}{lccc}
\hline \multicolumn{1}{c}{ Characteristic } & Partial resection $(\mathrm{n}=29)$ & Subtotal resection $(\mathrm{n}=26)$ & $\mathrm{p}$-value \\
\hline Age $(\mathrm{yr})$ & $32.5 \pm 12.33(18-57)$ & $31.7 \pm 12.62(14-61)$ & $0.825^{\mathrm{a})}$ \\
Follow-up duration (mo) & $32.5 \pm 11.54(29-49)$ & $34.6 \pm 11.50(24-44)$ & $0.495^{\mathrm{a})}$ \\
Site & $16(55.2)$ & $15(57.7)$ & $1.000^{\mathrm{b})}$ \\
Right & $13(44.8)$ & $11(42.35)$ & \\
Left & & $14(53.8)$ & $1.000^{\mathrm{b})}$ \\
Sex & $15(51.7)$ & $12(46.2)$ & $22(84.6)$ \\
Male & $14(48.3)$ & $20(76.9)$ & $1.000^{\mathrm{b})}$ \\
Female & $25(86.2)$ & $23(88.5)$ & $0.764^{\mathrm{b})}$ \\
Positive Hoffa's test & $21(72.4)$ & & $1.000^{\mathrm{b})}$ \\
Positive findings on MRI & $26(89.7)$ & & \\
Positive lidocaine test & & & \\
\hline
\end{tabular}

Values are presented as mean \pm standard deviation (range) or number (\%).

MRI: magnetic resonance imaging.

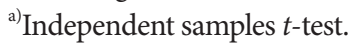

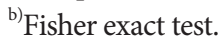


Severe pain, an antalgic block, and defensive behavior are considered diagnostic of fat pad impingement. In our cohort, Hoffa's test was positive in $86.2 \%$ of patients in the partial resection group and $84.6 \%$ of patients in the subtotal resection group.

MRI is also a useful means of diagnosing fat pad impingement and other concomitant pathologies. Hypertrophy and localized edema in the superior and posterior parts of the fat pad, a fluidfilled infrapatellar bursa, horizontal and vertical clefts, fibrosis, and calcifications may be observed ${ }^{11,18)}$. We identified inflammation of the IFP in some cases but treated only impingement of the

Table 2. Comparison of Preoperative Status and Final Follow-up Clinical Outcomes

\begin{tabular}{lccc}
\hline \multicolumn{1}{c}{ Variable } & $\begin{array}{c}\text { Partial } \\
\text { resection }\end{array}$ & $\begin{array}{c}\text { Subtotal } \\
\text { resection }\end{array}$ & p-value $^{\text {a) }}$ \\
\hline Visual analog scale & $5.6 \pm 0.61$ & $5.7 \pm 0.43$ & 0.425 \\
Preoperative & $1.4 \pm 0.63$ & $1.8 \pm 0.77$ & 0.040 \\
Final follow-up & $<0.001$ & $<0.001$ & \\
p-value & & & \\
IKDC 2000 & $47.5 \pm 7.41$ & $47.7 \pm 9.09$ & 0.918 \\
Preoperative & $70.9 \pm 6.15$ & $71.9 \pm 9.33$ & 0.644 \\
Final follow-up & $<0.001$ & $<0.001$ & \\
p-value & & & \\
Lysholm & & & \\
Preoperative & $42.5 \pm 7.17$ & $45.2 \pm 4.18$ & 0.100 \\
Final follow-up $_{\text {p-value }}^{\text {b) }}$ & $82.2 \pm 7.61$ & $82.3 \pm 6.01$ & 0.970 \\
Postoperative complications & No & $<0.001$ & \\
\hline
\end{tabular}

Values are presented as mean \pm standard deviation.

IKDC: International Knee Documentation Committee.

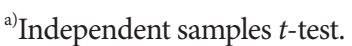

${ }^{\text {b) }}$ Paired samples $t$-test.
IFP between the patella and femoral trochlea as a diagnostic finding (Fig. 6). The main role of MRI is to exclude other disorders of the knee joint. We observed IFP impingement on MRI in $72.4 \%$ of patients in the partial resection group and $76.9 \%$ of patients in the subtotal resection group. We also used direct injection of lidocaine into the IFP to distinguish intra-articular lesions from extra-articular lesions ${ }^{17}$, a test that was positive in $89.7 \%$ of patients in the partial resection group and $88.5 \%$ of patients in the subtotal resection group.

We were unable to identify IFP impingement on MRI in 27.6\% of patients in the partial resection group and $23.1 \%$ of patients in the subtotal resection group. However, Hoffa's test and the outcome of lidocaine infiltration were positive in these patients, and all complained of persistent anterior knee pain. In these cases, we undertook a diagnostic arthroscopy and made a therapeutic intervention when we found a "tongue-like protrusion" of the IFP that had not been observed on MRI. Clinicians should be mindful of IFP impingement when investigating patients with persistent and unexplained anterior knee pain.

It has been reported that arthroscopic resection of the fat pad is an effective treatment for infrapatellar impingement. Kumar and colleagues found that fat pad resection improved knee function significantly, as measured using the Lysholm score, from 37.4 preoperatively to 90.8 at the final follow-up, and concluded that arthroscopic resection of the impinging part of the fat pad achieved sustained relief of symptoms with low rates of morbidity and complications ${ }^{11)}$. Ogilvie-Harris and Giddins also reported significant improvements in symptoms and function after resection of the fat pad at a mean follow-up of 76 months ${ }^{12)}$. We also documented statistically significant improvements in knee pain and function after partial and subtotal arthroscopic fat pad resection, but there was no significant difference in clinical outcome between the groups; therefore, partial resection was as effective as
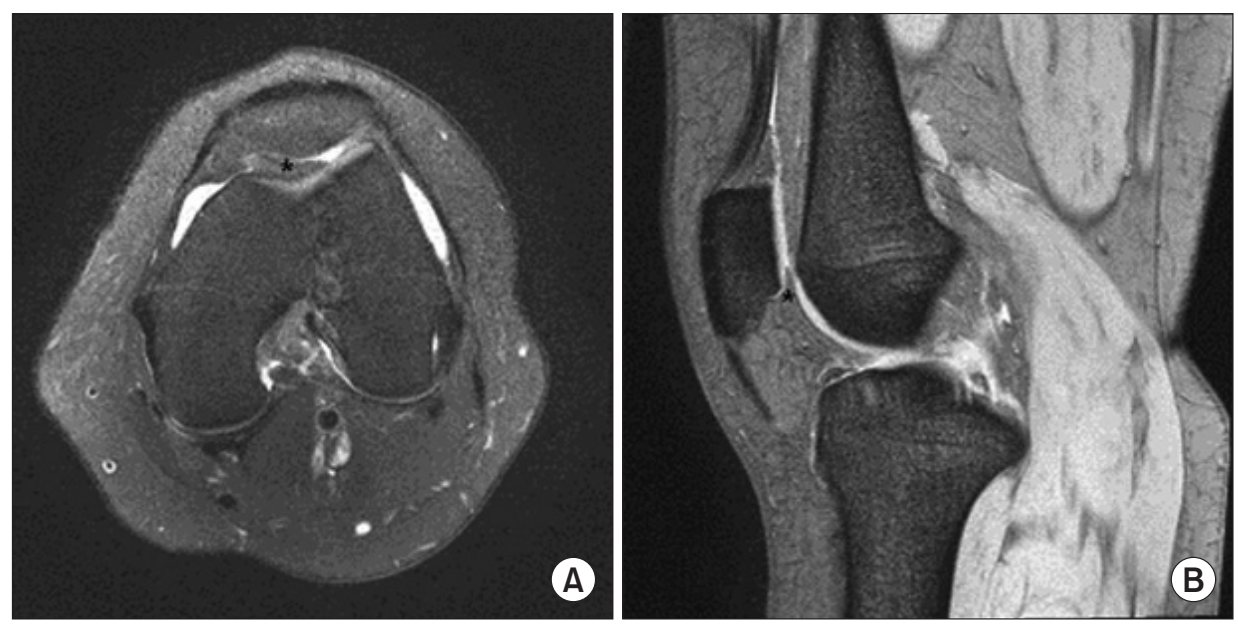

Fig. 6. T2-weighted turbo spin-echo magnetic resonance imaging of a 30 -year-old female with impingement of infrapatellar fat pad between the patella and femoral trochlea, indicated by the asterisk. (A) Axial image. (B) Sagittal image. 
subtotal resection.

The IFP maintains the blood supply to adjacent structures, such as the anterior cruciate ligament, so preservation of the fat pad should be a priority when planning surgical procedures ${ }^{19-21)}$. Total resection of the IFP influences patellar biomechanics and knee kinematics ${ }^{22)}$. Liu and colleagues emphasized the importance of preserving as much of the normal IFP as possible due to its tendon-repairing capabilities, thereby potentially decreasing the incidence of patella baja ${ }^{23)}$. We encountered no postoperative complications after fat pad resection in either group, likely because partial resection maximizes fat pad function and subtotal resection preserves at least some fat pad function. We think that partial resection is sufficient to relieve the impingement symptoms and that total resection should be avoided to retain a physiological fat pad volume.

Our study had some limitations. First, the sample size was relatively small, so some statistically and clinically significant findings may have been missed. Second, our positive MRI findings may have been subjective. However, we confirmed the findings during the arthroscopic surgery. Third, long-term outcome data may be needed to distinguish the differences between partial and subtotal resection.

\section{Conclusions}

In conclusion, arthroscopic resection for IFP impingement between the patella and femoral trochlea resulted in favorable clinical outcomes, regardless of whether the resection was partial or subtotal. Thus, partial resection can be an appropriate treatment for IFP impingement between the patella and femoral trochlea, considering partial resection was as effective as a subtotal resection and preserved more of the fat pad.

\section{Conflict of Interest}

No potential conflict of interest relevant to this article was reported.

\section{References}

1. Hoffa A. The influence of the adipose tissue with regard to the pathology of the knee joint. JAMA. 1904;43:795-6.

2. Saddik D, McNally EG, Richardson M. MRI of Hoffa's fat pad. Skeletal Radiol. 2004;33:433-44.

3. von Engelhardt LV, Tokmakidis E, Lahner M, David A, Haage P, Bouillon B, Lichtinger TK. Hoffa's fat pad impinge- ment treated arthroscopically: related findings on preoperative MRI in a case series of 62 patients. Arch Orthop Trauma Surg. 2010;130:1041-51.

4. Dye SF, Vaupel GL, Dye CC. Conscious neurosensory mapping of the internal structures of the human knee without intraarticular anesthesia. Am J Sports Med. 1998;26:773-7.

5. Biedert RM, Sanchis-Alfonso V. Sources of anterior knee pain. Clin Sports Med. 2002;21:335-47.

6. Duri ZA, Aichroth PM, Dowd G. The fat pad: clinical observations. Am J Knee Surg. 1996;9:55-66.

7. Magi M, Branca A, Bucca C, Langerame V. Hoffa disease. Ital J Orthop Traumatol. 1991;17:211-6.

8. Crossley K, Bennell K, Green S, Cowan S, McConnell J. Physical therapy for patellofemoral pain: a randomized, double-blinded, placebo-controlled trial. Am J Sports Med. 2002;30:857-65.

9. McConnell J. The physical therapist's approach to patellofemoral disorders. Clin Sports Med. 2002;21:363-87.

10. Metheny JA, Mayor MB. Hoffa disease: chronic impingement of the infra-patellar fat pad. Am J Knee Surg. 1988;1:134-9.

11. Kumar D, Alvand A, Beacon JP. Impingement of infrapatellar fat pad (Hoffa's disease): results of high-portal arthroscopic resection. Arthroscopy. 2007;23:1180-6.

12. Ogilvie-Harris DJ, Giddens J. Hoffa's disease: arthroscopic resection of the infrapatellar fat pad. Arthroscopy. 1994;10:184-7.

13. Morini G, Chiodi E, Centanni F, Gattazzo D. Hoffa's disease of the adipose pad: magnetic resonance versus surgical findings. Radiol Med. 1998;95:278-85.

14. Emad Y, Ragab Y. Liposynovitis prepatellaris in athletic runner (Hoffa's syndrome): case report and review of the literature. Clin Rheumatol. 2007;26:1201-3.

15. Carlsson AM. Assessment of chronic pain. I: aspects of the reliability and validity of the visual analogue scale. Pain. 1983;16:87-101.

16. Irrgang JJ, Anderson AF, Boland AL, Harner CD, Kurosaka M, Neyret P, Richmond JC, Shelborne KD. Development and validation of the international knee documentation committee subjective knee form. Am J Sports Med. 2001;29:600-13.

17. Lysholm J, Gillquist J. Evaluation of knee ligament surgery results with special emphasis on use of a scoring scale. Am J Sports Med. 1982;10:150-4.

18. Patel SJ, Kaplan PA, Dussault RG, Kahler DM. Anatomy and clinical significance of the horizontal cleft in the infrapatellar fat pad of the knee: MR imaging. AJR Am J Roentgenol. 
1998;170:1551-5.

19. Doner GP, Noyes FR. Arthroscopic resection of fat pad lesions and infrapatellar contractures. Arthrosc Tech. 2014;3:e413-6.

20. Gallagher J, Tierney P, Murray P, O'Brien M. The infrapatellar fat pad: anatomy and clinical correlations. Knee Surg Sports Traumatol Arthrosc. 2005;13:268-72.

21. Huberti HH, Hayes WC. Patellofemoral contact pressures: the influence of q-angle and tendofemoral contact. J Bone
Joint Surg Am. 1984;66:715-24.

22. Bohnsack M, Wilharm A, Hurschler C, Rühmann O, Stukenborg-Colsman C, Wirth CJ. Biomechanical and kinematic influences of a total infrapatellar fat pad resection on the knee. Am J Sports Med. 2004;32:1873-80.

23. Liu YP, Li SZ, Yuan F, Xia J, Yu X, Liu X, Yu GR. Infrapatellar fat pad may be with tendon repairing ability and closely related with the developing process of patella Baja. Med Hypotheses. 2011;77:620-3. 